\title{
Assembly strategy of liposome and polymer systems for siRNA delivery
}

\author{
Huiling Song ${ }^{\mathrm{a}}$, Stephen L. Hart ${ }^{\mathrm{b}}$, Zixiu Du ${ }^{\mathrm{a}, *}$
}

${ }^{a}$ Engineering Research Center of Cell \& Therapeutic Antibody, Ministry of Education, and School of Pharmacy, Shanghai Jiao Tong University, 800 Dongchuan Road, Shanghai 200240, P.R. China

${ }^{\mathrm{b}}$ Department of Genetics and Genomic Medicine, UCL Great Ormond Street Institute of Child Health, 30 Guilford Street, London WC1N 1EH

*Correspondences: Zixiu Du, Tel. +82 34204739, Fax +82 34204457, E-mail: zixiudu@sjtu.edu.cn.

\begin{abstract}
In recent years, gene therapy has made tremendous progress in the development of disease treatment. Among them, siRNA offers specificity of gene silencing, ease of synthesis, and short development period, and has been intensively studied worldwide. However, siRNA as the hydrophilic polyanion is easily degraded in vivo and poorly taken up into cells and so, the benefits of its powerful gene silencing ability will not be realized until better carriers are developed that are capable of protecting siRNA and delivering it intact to the cytoplasm of the target cells. Cationic liposomes (CLs) and cationic polymers (CPs) are the main non-viral siRNA vectors, there have been a lot of reports on the use of these two carriers to deliver siRNA. Whereas, as far as we know, there have been few review articles that provide an indepth summary of the siRNA loading principle and internal structures of the siRNA delivery system. We summarize in detail the formation principle and assembly structure of the cationic liposome-siRNA and polymer-siRNA complexes, and point out their advantages and characteristics and also show how to perfect their assembly and improve their clinical application in the future. It supports some useful suggestions for siRNA therapy, specifically, safe and efficient delivery.
\end{abstract}

Key words: siRNA delivery system, Cationic liposome, Cationic polymer, Targeted 
delivery, Silence efficiency 


\section{Introduction}

With the rapid development of modern biotechnology, gene therapy has shown tremendous potential in the diagnosis and treatment of severe diseases. Compared with traditional small molecule drugs, the specificity of gene therapy offers great advantages. So far, the research of gene drugs mainly focuses on plasmid DNA (pDNA), messenger RNA (mRNA), and RNA interference (RNAi). Among them, short interfering RNA (siRNA) is easy to synthesize and is less likely to suffer from drug resistance. Not only does it offer greater specificity than microRNA (miRNA), but also it needs only to be delivered to the cytoplasm compared with pDNA, which must first be transcribed in the nucleus [1-3]. When it enters the cytoplasm, it combines with mRNA in the form of a single strand to generate a RISC complex, and silence the target gene specifically [2]. Thus, siRNA can, in theory, silence any gene and is expected to be one of the most effective treatments for many diseases.

However, due to the nature of the nucleic acid molecules, the application of siRNA remains limited. Firstly, the extracellular instability of siRNA leads to rapid degradation before reaching the target site. Secondly, hydrophilic, negatively-charged nucleic acids cannot cross the cell membrane. Furthermore, successful release of siRNA into the cytoplasm after entering the cell is also a key factor for its efficacy [3]. Therefore, it is important to ensure the successful delivery of siRNA by vectors.

Early vectors for gene therapy were mainly derived from viruses. Genes can be transferred effectively into mammalian cells through viruses achieving gene expression. Although viral vectors have extraordinary ability in gene transfection, their safety has always been a cause for concern, such as carcinogenic effects, immunogenicity, mutations, etc. $[4,5]$. In 1999, an adenoviral vector caused the death of a patient in a trial for gene therapy of ornithine transcarbamylase by intravenous administration of a 
relatively high dose of the virus, which raised concerns over the safety of viral vectors for other applications [6]. In addition, the limited loading capacity and difficulties in synthesis at larger scale of viral vectors, such as adeno-associated virus (AAV) and lentiviral vectors, also restricts their clinical application [7-11]. In contrast, non-viral vectors are much safer and easier to synthesize and modify, and have become the driving force for the ongoing development fo siRNA therapies. The first siRNA drug on the market is Patisiran which delivered by a lipid-based carrier, indicating the usefulness of non-viral vectors in siRNA delivery [12] whereasthey were less effective for pDNA-based gene delivery for reasons described[13]. In order to overcome in vivo biological barriers and to improve transfection efficiency of siRNA, liposomes and polymers, as the main components of non-viral vectors for siRNA delivery, have been greatly modified and complexed in the past decades.

\section{The classification and design strategy of siRNA delivery systems}

Currentl, carriers formed by liposomes and polymers are mainly divided into three categories: cationic liposomes (CL), cationic polymers (CP), and the combination of liposomes and polymers (LP). Here, we mainly review the internal structure of the above siRNA delivery systems, to investigate the relationship between the delivery efficiency and assembly structure of lipoplexes, polyplexes and lipopolyplexes.

\subsection{Cationic Liposome-siRNA complexes}

\subsubsection{The formulations of cationic Liposome-siRNA complexes}

Among the siRNA delivery systems, liposomes composed of phospholipid bilayers are most widely used. As we know, siRNAs are negatively charged, so it is reasonable to consider using CLs to absorb siRNA through electrostatic interactions, 
which is why many studies deliver siRNAs as complexes with liposomes in vitro[14]. There are various CLs for siRNA delivery, such as the classic lipids 1,2-Dioleoyl-3trimethylammonium-propane (DOTAP) and 1,2-di-O-octadecenyl-3trimethylammonium propane (DOTMA), which are combined with neutral lipids such as1,2-dioleoyl-sn-glycero-3-phosphocholine (DOPC) and 1,2-dioleoylsn-glycero-3phosphatidyl-ethanolamine (DOPE), (Figure 1). Positively charged CLs are conducive to transmembrane transport. Of course, this is a double-edged sword, as CLs also interact with proteins in the blood and, as a result, are cleared quickly from the circulation andrelease siRNA immediately on contact with human serum[15]. Furthermore, cationic lipids can display cytotoxicity, such as destruction of the cell membrane and by generating reactive oxygen species [16].

In contrast, unlike cationic lipids, anionic lipids are less immunogenic [17]. Many researchers have begun to switch from using cationic lipids to anionic lipids in siRNAloaded liposomes. In fact, the delivery of anionic lipids is less efficient, because the electrostatic repulsion between anionic substances and siRNA prevents them from being compacted stably, thus cations have to be incorporated into the system to enable the condensation of siRNA[18], for example, $\mathrm{Ca}^{2+}$ can be utilized to help anionic lipids to wrap siRNA, whereas the anionic lipoplexes have a poor transmembrane ability compared to CLs.

As there are lots of problems with charged liposome, drug-loaded neutral liposomes have been investigated to reduce the toxicity associated with CLs [15]. Theydo not suffer from electrostatic repulsion at the cell membrane as occurswith anionic liposomes. To further optimize the formulation of liposomes, Artur CavacoPaulo et al., used DOPE-derived neutral liposomes to encapsulate siRNA [15]. In this neutral liposome preparation, siRNA molecules are encapsulated within the core of highly PEGylated liposomes, so there is no siRNA binding to the outer surface of the 
liposome which largely avoids the situation where the siRNA competes with the negatively charged serum components leading to its release and degradation in the serum [15]. Thus, when designing siRNA lipid formulations, it is necessary to balance the ratio ofpositive and negative ions to reduce the toxic and side effects of the carriers and improve the therapeutic effects of the drugs.

To increase the stability of lipid-based carriers, more stable lipid analogs are being studied deeply. In general, the structure of lipid analogs includes a positively charged hydrophilic head, a linking moiety, and two hydrophobic chains such as L319a representative molecule (Figure 1). The biggest feature of L319 is the ester structure in the carbon chain instead of carbon-carbon double bonds. Since the ester group is similar to the carbon-carbon double bond, on the basis of retaining the space between the two alkyl chains, the toxicity of the carrier is reduced due to the biodegradability of the ester group. During the screening process to identify L319, it was found that the position of the ester group also had a great influence on the molecular efficiency with a preferred location in the middle of the alkyl chain forgreater efficacy [19]. On this basis, amino glycosides such as arginine can also be used to replace the amino head [20].

Another representative structure is C12-200 (Figure 1), which, in contrast to L319, contains more than one amine center, and hydrophobic chains connected by a carbonnitrogen single bond formed between epoxide and amine group. It was reported that such molecules require a hydrophobic chain of medium length, because shorter ones lead to reduced efficiency while those with longer chains are more difficult to dissolve in the preparation process [21].

In short, the basic structure of this type of carrier is an amphiphilic molecule consisting of a hydrophilic head and a hydrophobic chain containing an ionizable amino group. Current studies have found that there is no absolute correlation between the 
results of in vitro experiments and in vivo experiments of carriers. Therefore, seeking the relation between molecular structure and carrier efficacy has become one of the main projects of current research. The research from Robert Langer's team found that a lipid derivative carrier with good activity must meet the following requirements: The amines must contain at least one tertiary amine group; the hydrophobic chains must be of appropriate length; the particle size must be maintained at 50 to $100 \mathrm{~nm}$; the value of pKa must lie in 6.2 to 6.5 [22]. According to these principles, they designed many carriers, and some of them have promising efficiency, supporting these rules.

The use of lipids for siRNA delivery was approved in 2018, with Patisiran, the world's first siRNA drug, using the lipid derivative DLin-MC3-DMA as the carrier, marking the success of lipid derivatives as siRNA carriers. However, there are still many deficiencies in lipid-derived carriers, such as instability and safety concerns that need to be addressed.

\subsubsection{The structure of cationic liposome-siRNA complexes}

Generally, cationic liposomes comprise cationic lipids and helper lipids (neutral lipids). For example, DOTAP is combined with DOPC or DOPE to form CLs. The structure of lipoplexes formed by DOTAP/DOPC is a multilayered sandwich with siRNA between the cationic lipids (lamellar complexes, $\mathrm{La}^{\mathrm{C}}$ ), while that of lipoplexes formed by DOTAP/DOPE and siRNA tends to transit from $\mathrm{La}_{\mathrm{a}}^{\mathrm{C}}$ to the isotropic/inverted hexagonal configuration $\left(\mathrm{H}_{\mathrm{II}}{ }^{\mathrm{C}}\right)$. The key reason for the difference between the above structures is the head space steric hindrance of DOPC and DOPE[23]. As we can see, the cross-sectional area of the combined headgroup of DOTAP/DOPC in lipoplexes is comparable with that of the hydrophobic areas in isolation, while the cross-sectional area of the combined headgroup of DOTAP/DOPE is less than that of the hydrophobic areas in isolation (Figure 2). Therefore, the lipoplexes adopt a molecular 'cone' shape, 
which promotes the formation of inverted, non-bilayer phases such as the hexagonal $\mathrm{H}_{\text {II }}{ }^{\mathrm{C}}$ phase. It was demonstrated that the helper lipids affect the self-assembly structures of the complexes formed by cationic liposome and siRNA. There have been many siRNA delivery systems like the above typical examples of DOTAP/DOPC and DOTAP/DOPE vectors. DOPE enables membrane fusion[24], since the hydrophobic headgroup cross-sectional area of DOPE is less than that of hydrocarbon chain domains, the addition of DOPE to DOTAP promotes the siRNA lipoplex structure changes from $\mathrm{La}^{\mathrm{C}}$ into $\mathrm{H}_{\mathrm{II}}^{\mathrm{C}}$ phase[18], making the compound have good meltability and significantly improve transmembrane efficiency[25]. In addition to the membrane fusion, DOPE possesses a highly effective delivery capability in vitro, but the stability of $\mathrm{H}_{\mathrm{II}}^{\mathrm{C}}$ structure is not as good as that of $\mathrm{La}^{\mathrm{C}}$ in vivo, which leads to rapidly reduced delivery. DOPC and cholesterol were always used as the helper to assistant CLs for siRNA package, and help to maintain the bilayer structure[26].

In order to achieve in vivo long-tern circulation and improve stability by avoiding aggregation and accumulation in the target tissue, CLs were PEGylated[27] and targeting moieties were anchored the on the surfaces of lipoplexes[28]. The advantages and dilemma of PEGylation of CLs in the overcoming the biological barriers in vivo have been discussed in a number of excellent reviews [28, 29]. In this review, we would like to emphasize the internal structure of nanocarriers in the PEGylation of liposomal siRNA delivery system. Since the PEGylated liposomes were prepared through thin-film hydration [30], ethanol injection methods [31] or postinsertion technology of PEGylation [32], except for post-insertion of PEGylation, the lipoplexes prepared through the other two preparation methods showed that the PEG domains of lipoplexes were located at both the internal and outside surface of liposomal membranes, which might slightly reduce the binding siRNA ability of CLs. 


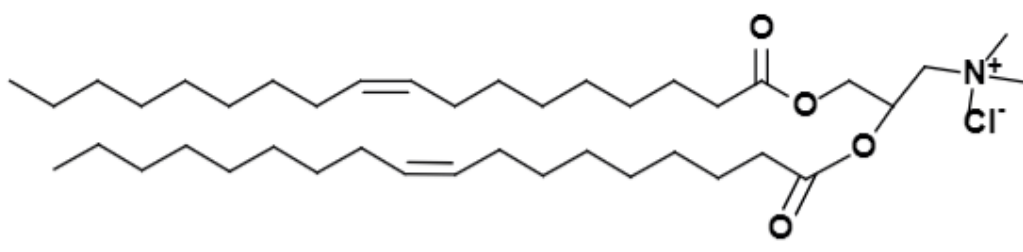

DOTAP

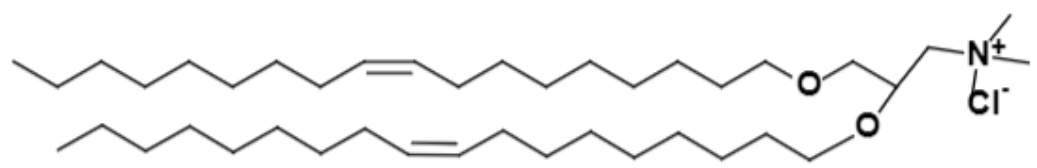

DOTMA

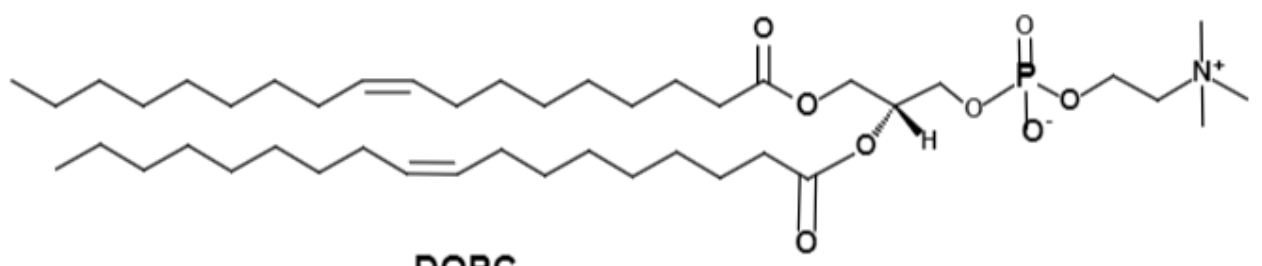

DOPC

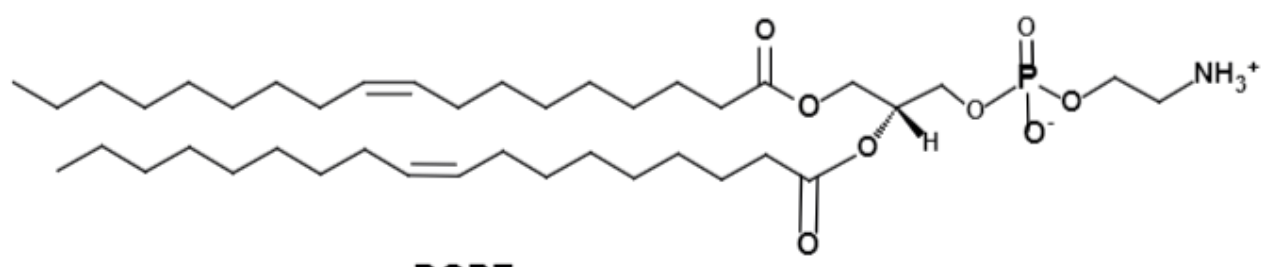

DOPE<smiles></smiles>

\section{L319}<smiles>CCCCCCCCCCCCCCCCC(O)CN(CCN1CCN(CCN(CCN(CC(O)CCCCCCCCC)CC(O)CCCCCCCCC)CC(O)CCCCCCCCC)CC1)CC(O)CCCCCCCCCC</smiles>

C12-200

Figure 1 Chemical structures of DOTAP, DOTMA, DOPC, DOPE, L319 and C12-200. 

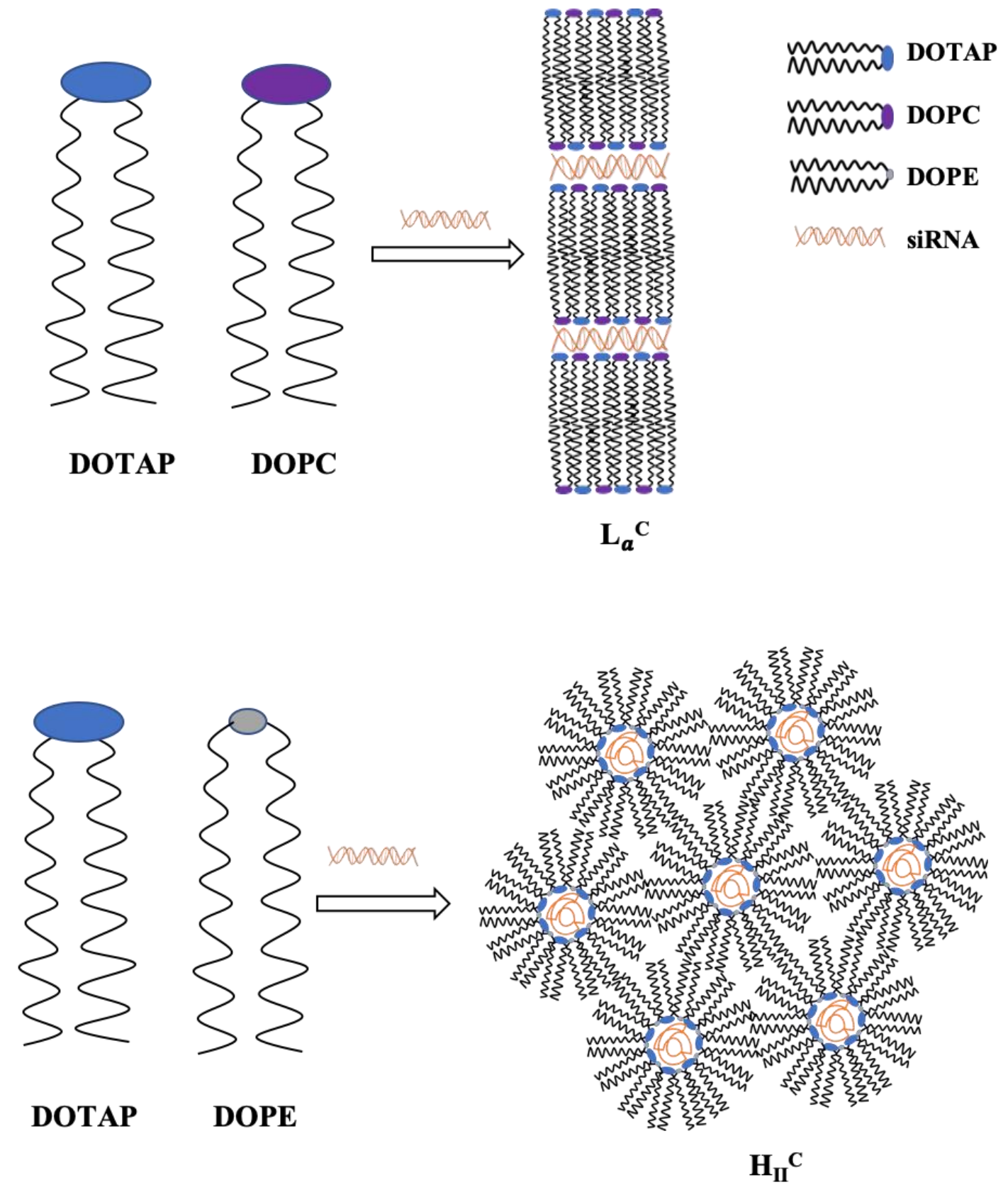

Figure 2 Schematic depiction of lipoplexes formed by DOTAP/DOPC and DOTAP/DOPE complexed with siRNA through static electricity.

\subsection{Cationic polymer-siRNA complexes}

CP can also wrap siRNA through electrostatic interaction. Compared to lipids, polymers are much more stable, easier to be synthesized and produced at large-scale 
[33] and have been widely studied. Compared to the CLs, polymers only depend on the strength of non-covalent interactions, such as electrostatic effects and hydrogen bonding to compress siRNA.

\subsubsection{The formulations of cationic polymer -siRNA complexes}

There have been many CPs utilized for siRNA delivery, all of which share common physiochemical characteristics, such as cationic charge, amphiphilicity, and fusogenicity. The representative polycations are polyethylenimine (PEI), poly L-Lysine (PLL), poly( $\beta$-amino esters) (PBAE), Poly (amido amine) (PAMAM). PEI is the most widely used material and offers a strong positive-charge to encapsulate siRNA into nanoparticle structures through electrostatic interactions, while its buffering capacity allows it to escape from the endosome after entering the cell [33]. Among them, 25kd PEI has high transfection efficiency and is widely used as a gold standard for polymer carriers. However, PEI is undegradable and its aggregation in the body may cause toxicity including embolism[34]. Poly-L - lysine (PLL) is a type of polyamino acid molecule which offers better biocompatibility than PEI. However, in vitro studies showed, PLL is less efficient at achieving siRNA-mediated gene silencing than PEI [35]. PBAE, a block copolymer produced by the reaction between bisacrylates and amines contains multiple-ester bonds and so PBAE is more biodegradable than PEI. However, PBAE is also less stable in the blood because of this biodegradability. PAMAM is a dendritic polymer characterized by the presence of a large number of ionizable end groups, which means they can effectively bind to a large amount of genetic material. These dendritic polymers have primary amine groups on the surface and tertiary amine groups inside. The negatively-charged genetic substances bind to primary amine groups on the carriers via electrostatic interactions, while the internal tertiary amino groups act as proton sponges in the endosome and enhance the endosomal escape in the cytoplasm [36]. Highly branched PAMAMs provide efficient 
gene transfection but are highly toxic due to their high cationic charge [37]. Over time, the characteristics of cationic polymers have been optimized from biosafety, biocompatibility, biodegradation, $\mathrm{pH}$ sensitivity to degradation products. Many novel biomaterials have been designed and prepared for safe and high transfection efficiency althoughthe chemical toxicity of CPs has to be considered. To reduce toxicity of carriers and improve the biocompatibility, endogenous substances were explored as potential materials for siRNA delivery, such as carbohydrates and poly-amino acids [38, 39]. Du $\mathrm{Z}$ et al designed polycation gene carriers with the endogenous molecule spermine as the basic unit structure, which can be degraded to spermine which were shown to be less toxic[40, 41]. From a structural point of view, linear CPs with higher molecular weight usually have stronger transfer efficiency, but are also more toxic. By quaternization of their amine groups, the stability of binding of siRNA to carrier materials can be increased and the transfection efficiency also can be further enhanced [42]. As for branched CPs, its three-dimensional structure can better protect siRNA and can form a wider range of carrier with different properties than linear chain, due to its multifunctional terminal groups (Figure 3). Another critical factor for the efficiency of CP-based siRNA transfection is the presence of tertiary amino groups in the pKa range from 5 to 7 which is the marker of the proton sponge effect, necessary for endosomal escape[43]. 


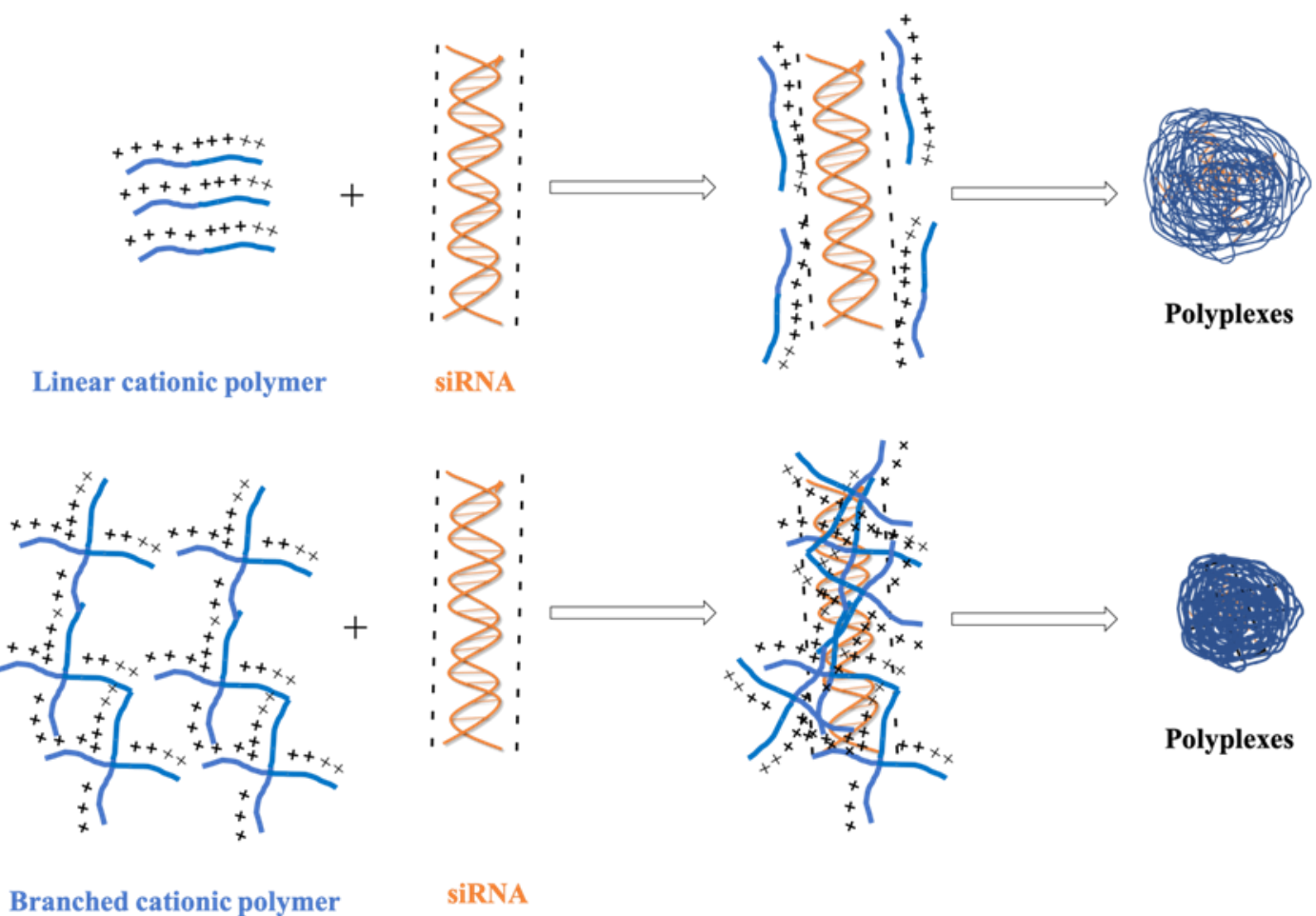

Figure 3 Schematic depiction of polyplexes formed by Linear and branched CPs and siRNA.

Similar to PEGylated CLs, PEGylation of CPs enhances the stability of the gene delivery system in the body increasing the circulation time of polyplexes in the blood and drug accumulation at tumour sites[44]. At the same time, there is a dilemma in PEGylation of polyplexes. Amphiphilic block copolymers can form micelles easily and generate smaller sized complexes with higher particle density than CPs without hydrophobic segments. For example, polycaprolactone (PCL) can be introduced to the PEG-PLL-siRNA, and so enabling endocytic internalization of PCL-containing carriers leading to higher siRNA delivery and gene silencing efficiency. On the other hand, novel PEG-containing CPs are needed whereby the attached PEG is removed following endocytosis via broken connection[45]. In order to reduce the blood clearance and accumulationof siRNA in the tumour, it is necessary to optimize the $\mathrm{CP}$ carriers to improve targeting ability and decrease adverse effects. The targeting moieties were 
anchored at the surfaces of the polyplexes through chemical conjugation with PEG to achieve active targeting delivery $[45,46]$ (Figure 4).

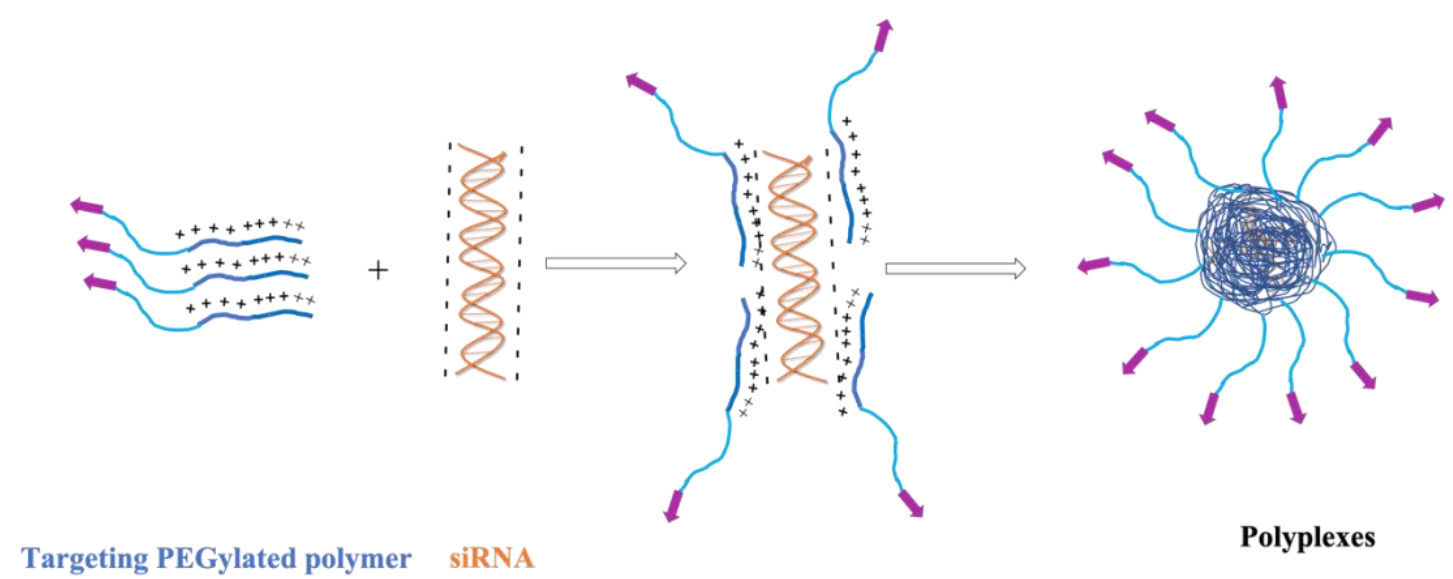

Figure 4 The formulation of targeting PEGylated polyplexes.

\subsubsection{The structure of cationic polymer -siRNA complexes}

So far, various CP-carriers have been used for siRNA delivery and they are easily prepared[47]. Unlike liposomes, which have good biocompatibility and can protect siRNA within the hydrophobic phospholipid membrane, polymer-based carriers are less capable of completely encapsulating siRNA through electrostatic interactions alone (Figure 3 and 4). Thus, in the circulation, the cargo easily leaks from the CP-siRNA complexes due to the small molecules in the bloodstreamentering the nanoparticles,even with PEGylation, which leads to the degradation of siRNA and hinders the delivery and therapeutic effect of siRNA in vivo. It has been shown that pKa and density of amines are the key factors for the packaging capability of polycationic vectors. CPs enable packaging of siRNA through electrostatic interactions in the environment where $\mathrm{pH}$ is lower than the corresponding $\mathrm{pKa}$ of amine groups. After the CP-siRNA complexes were endocytosed by the targeted cells, siRNA release was induced by the "proton sponge" effect and the degradation of vectors in the 
acidic conditions of the cytoplasm [48].

Actually, it is difficult to accurately control the targeting moiety grafting ratio, molecular weights and polydispersity of targeting modified PEGylated CPs through chemical synthesis, thus, the consistency and large-scale production of uniform formulation components are restricted. It is necessary to adopt effective strategies to overcome the above-mentioned drawbacks, so as to apply polyplexes to the clinic.

\subsection{Liposome-polymer-siRNA complexes (LPR)}

\subsubsection{The formulations of LPR}

With the development of siRNA therapy, siRNA delivery systems that selfassemble with both cationic polymers and liposomes (lipopolyplexes) display improved stability, increasing the drug loading and achieving more efficient delivery of siRNA in vivo. Leaf Huang et al. reported a tumor-targeted LPD formulation (liposomepolycation-DNA complex) for siRNA delivery [49-51], which has been the representative of liposome-polymer combined delivery for siRNA. Researchers also designed anionic liposome-siRNA complexes, which are formed by using cation polymers or polypeptides to bind siRNA before encapsulating it into anionic liposomes. Qiongfang $\mathrm{Yu}$ et al. combined siRNA with PEI and loaded it into anionic liposomes along with glycyrrhizic acid, which not only increased the solubility and anti-cancer effects of glycyrrhizic acid, but also reduced the toxicity of PEI-siRNA [52, 53]. In addition, Hart SL et al reported an RTN formulation (Receptor-targeted nanoparticles), which consists of a mixture of cationic, receptor-targeting peptides, CL and siRNA [54, 55]. It differs from the conventional LPD formulation in that the cationic peptide not only mediates packaging of siRNA but also contains a peptide targeting moiety for specific delivery. 
Based on the above two formulations, a variety of LPR have been reported [56], most of them exhibited excellent gene knockdown and transfection efficiencies, and enhanced the targeted delivery and self-assembly of LPR, while decreasing toxicity at the same time $[57,58]$.

\subsubsection{The structures of LPR}

The structure and self-assembly of LPD and LPR complexes have been discussed by Huang L et al, $[49,50]$. As shown in Figure 5, after the positively charged CL combined with the negatively-charged complexes through electrostatic interaction, the liposome collapses on the surface of the core formed by protamine and siRNA. Each liposome contains a single bilayer, therefore, there are two bilayer membranes on the surface of the negatively charged complexes after collapse. The outer bilayer (regular bilayer) is less stable due to the mutual electrostatic repulsion between the two bilayers whileDSPE-PEG can insert the re-assembly two bilayers easily, leaving only the inner bilayer on the surface. Inner bilayer as a supported bilayer is very stable which will not be dissolved by the micelles [49]. 


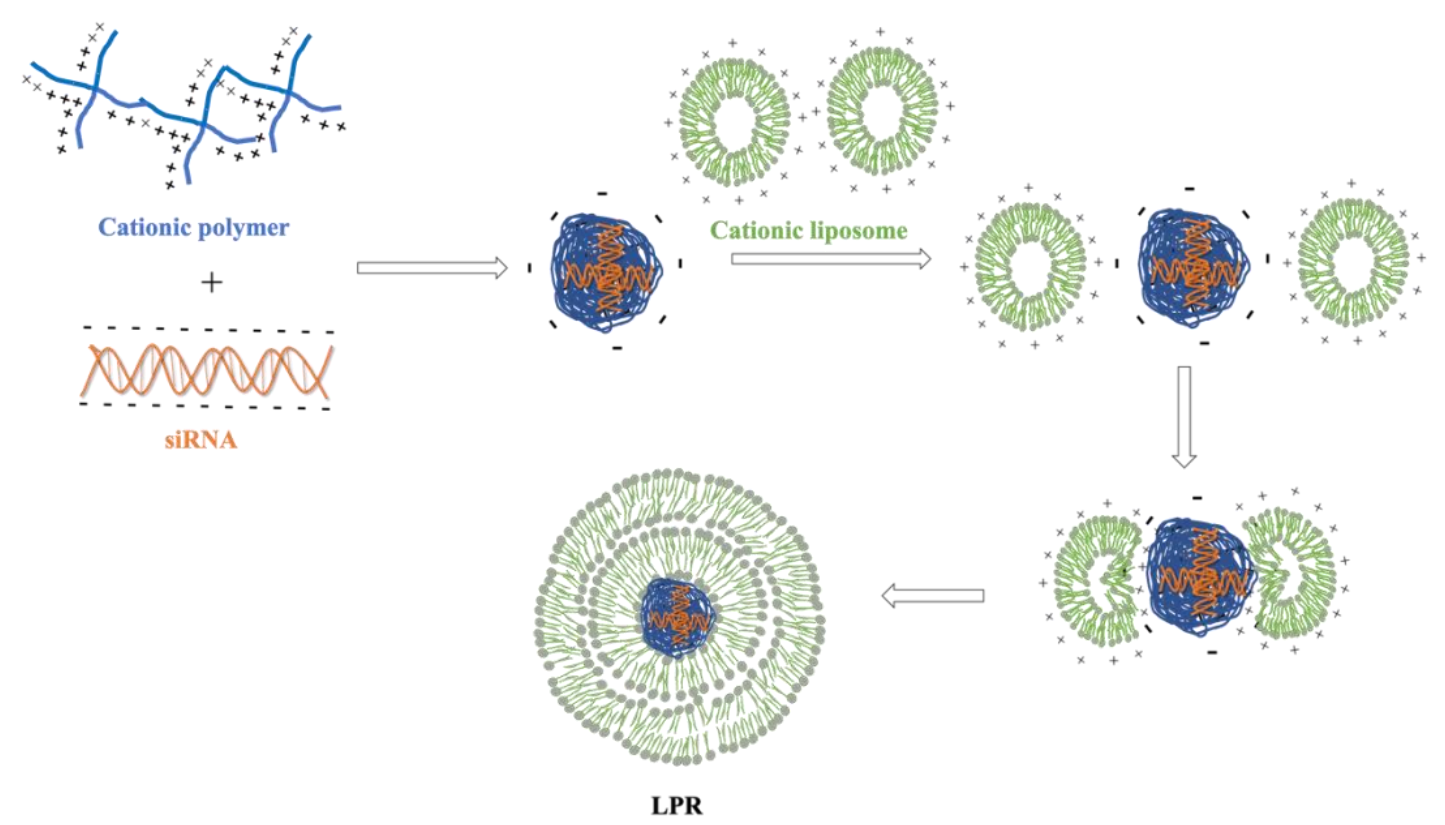

Figure 5 Illustration of the formation and structure of LPR.

On the other hand, the assembly and structure of RTNs are different from that of LPD, in which the peptide is designed for siRNA binding and targeting separated by a spacer domain, such as glycine-alanine (GA). In this case, the nucleic acid-binding element is a sequence of 16 lysine or arginine residues. The targeting element in the peptide is a neutral, integrin-targeting motif, which can be substituted for any other sequence of similar length. Typically, the cationic lipid is formulated into a CL with the neutral, fusogenic lipid DOPE firstly; then, the liposome is formulated into nanocomplexes on mixing with peptide and siRNA, at empirically optimized ratios. A single nanocomplex is shown with the proposed location of the three main residues (Figure 6). DOTAP/DOPE cationic liposome and peptides simultaneously performed electrostatic interaction with siRNA. Then, DOTAP/DOPE lipids rearrange and collapse on the complexes of peptide/siRNA. Finally, the three modular components assemble synergistically into a positive-charged ternary composite nanoparticle RNT. 


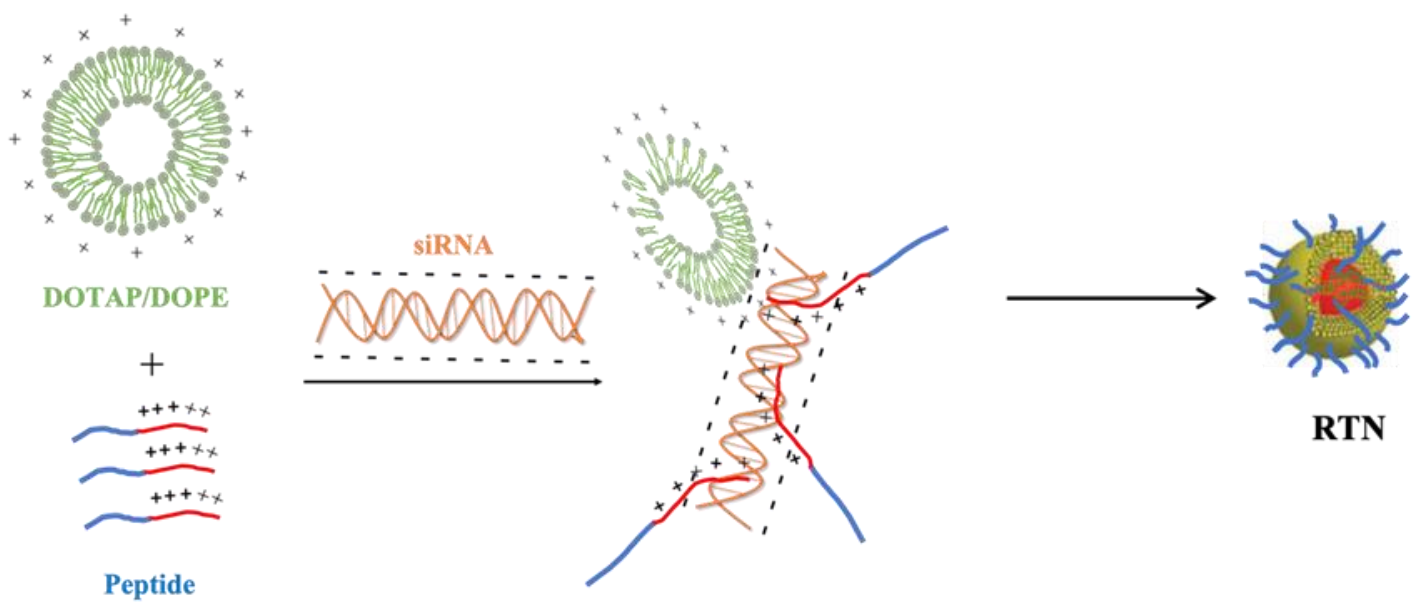

Figure 6 Illustration of the formation and structure of RTN.

\section{Stability strategy for siRNA delivery system}

In addition to modifying the mother structure of the carriers, attaching additional molecules onto the nanoparticles to improve stability is also a widely used strategy. Commonly used functional molecules to improve the stability of nanoparticles include polyethylene glycol (PEG), hyaluronic acid (HA), hydrophilic peptides and so on.

\subsection{PEG (Polyethylene glycol)}

PEG, as the most widely used substance in carrier design toimprove stability in vivo. But, infurther research, many problems have been exposed with the use of PEGPEG that cannot be ignored.

First and foremost is the PEG dilemma. When the carrier interacts with siRNA through electrostatic interactions, PEG shields the surface positive charge of siRNA complexes and hampers the interaction between the blood proteins and cargos. To date, most CL-siRNA and LPR complexes were PEG modified through inserting PEGylated 
lipids onto the bilayer membrane for in vivo delivery $[27,59]$. Compared to the CLsiRNA and LPR complexes, RTNs themselves contain targeting moieties, and these targeting moieties extend from the bilayer membrane. On the one hand, if lipids with long PEG were inserted into the bilayer membrane, the targeting function of RTNs may be compromised hindering the binding between nanoparticles and receptors on the cell surface [60].[61]. A nanoparticle composed of PEG, transferrin, and TNF-related apoptosis-inducing ligand (TRAIL) was reported. It is speculated that the reason for the reduced effectiveness of TRAIL may be related to the PEG [62]. Therefore, only lipids with short PEGs were used [60, 63, 64] (Figure 7).

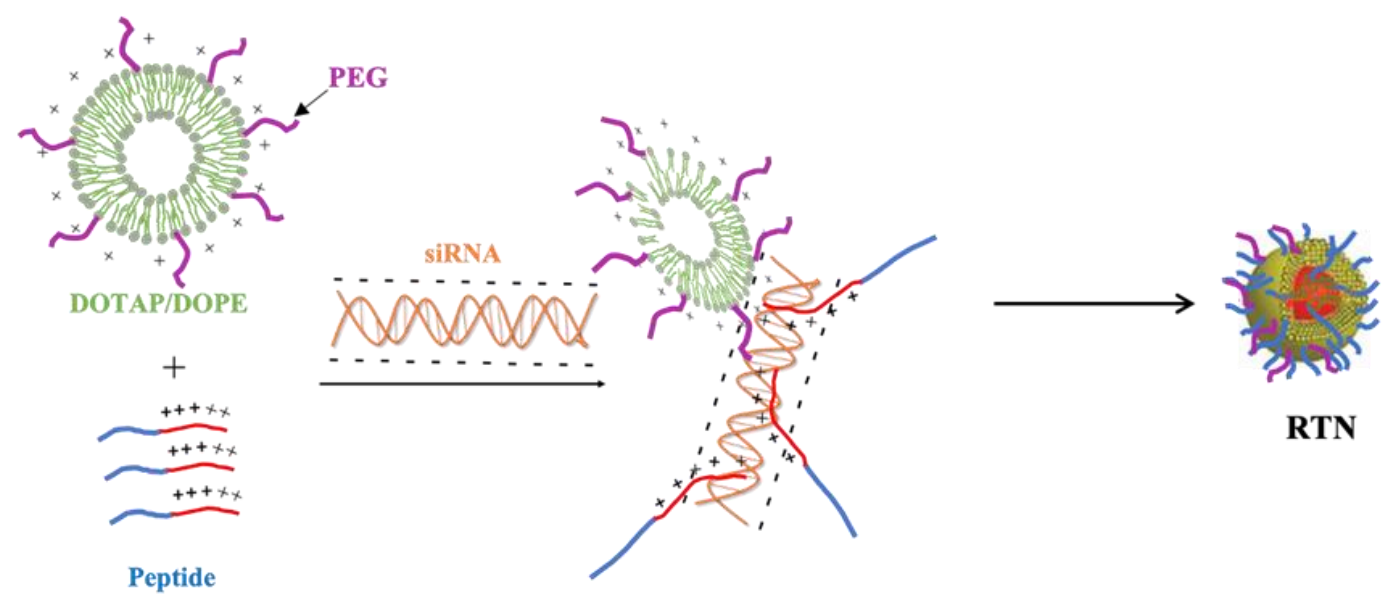

Figure 7 Illustration of the formation and structure of PEGylated RTN.

On the other hand, PEGylation of CP-siRNA complexes make the nanoparticles lose their compaction leading to a looser structure. Therefore, in this case, more polymers are required, which also increases its toxicity [65]. In the case of siRNAloaded chitosan, PEG was introduced to increase the water solubility of chitosan but this replaced the primary amino group, which was used for nucleic acid loading on the carrier, resulting in lower transfection efficiency[65]. Some studies have also shown that PEG increases particle size, which is not conducive to transfection [66]. Molecular weight, chain length, conformation, and coverage degree of PEG all have an effect on 
transfer efficiency, so these factors need to be considered carefully when we design the PEG-containing formulation. Unfortunately, there is no good method for accurate quantification of surface PEG so far, so it is difficult to establish the optimal amount of PEG in a formulation [67].

A further problem associated with PEGylation isaccelerated blood clearance $(\mathrm{ABC})$ in which, PEG can trigger an immune response after entering the body [68]. When the PEG-containing nanoparticles are injected for the first time, corresponding antibodies can be produced leading to rapid elimination of PEG nanoparticles after a second injection [69]. Based on these cases, a cleavable PEG linker was designed, which allows PEG to separate from the nanoparticles under certain intracellular stimuli after enabling the nanoparticles to complete in vivo circulation, and so enhancing their transfection efficiency [70-72]. Related experimental results showed that, in the group of PEGylated nanoparticles, the blood clearance rate was maintained at a constant rate, and no obvious $\mathrm{ABC}$ phenomenon was observed. Therefore, PEG is a double-edged sword and it is important to optimize the PEG ratio. Meanwhile, improved technologies for PEG quantification are urgently required

\subsubsection{Hyaluronic acid shielding}

Recently, Du ZX' team overcame the problems associated with PEG through using hyaluronic acid (HA) as the shielded shell.

Unlike PEG, HAis a common polysaccharide existing in the extracellular matrix. It is biocompatible and can be broken down by hyaluronidase in vivo. Most importantly, it does not produce an immune response [73]. The association of negatively charged HA with siRNA reduces the chance of siRNA being filtered by the glomerulus, because the negatively charged membrane of the glomerulus will repel it [74]. In addition to prolonging the blood circulation of the drug, it can target to $\mathrm{CD}_{44}$ specifically [75]. In 
our previous studies, we modified CP-siRNA complexes by chemical conjugating HA with CP. For example, HA-PEI was used to wrap siRNA and achieve in vivo targeted delivery [76-78]. To a certain extent, the conveying efficiency of HA-modified CPsiRNA complexes was improved, but the shortcomings of incomplete packaging of siRNA by the polymers remained. For PEGylated RTNs, the PEG chains and integrintargeting peptides protrude from the same disordered lipid layer. The PEG chains would weaken the targeting function of integrin-targeting peptides, and the optimized percentage of PEG and targeting moieties is difficult to control accurately. Thus, the pharmaceutical preparation of PEGylated RTNs is challenging to produce, indicating that PEGylation of RTNs would either reduce the delivery capability of siRNA vectors or incompletely shield the positive charge. In our previous study, HLPR was designed to utilize HA to shield the positive charge of the RTN[79, 80]. It indicated that HA could shield the positive charge of the ternary complexes, and did not impact on the targeting functions of the peptide (Figure 8). This study supported a novel and rational strategy for effective siRNA delivery in vivo. Compared with particles without HA, the HA-nanoparticles showed enhanced stability in blood $[79,80]$ and were less toxic than PEI25kDa and lipofectamine2000. The complex entered the cells through caveolamediated endocytosis preferentially, avoiding lysosomal degradation[81].

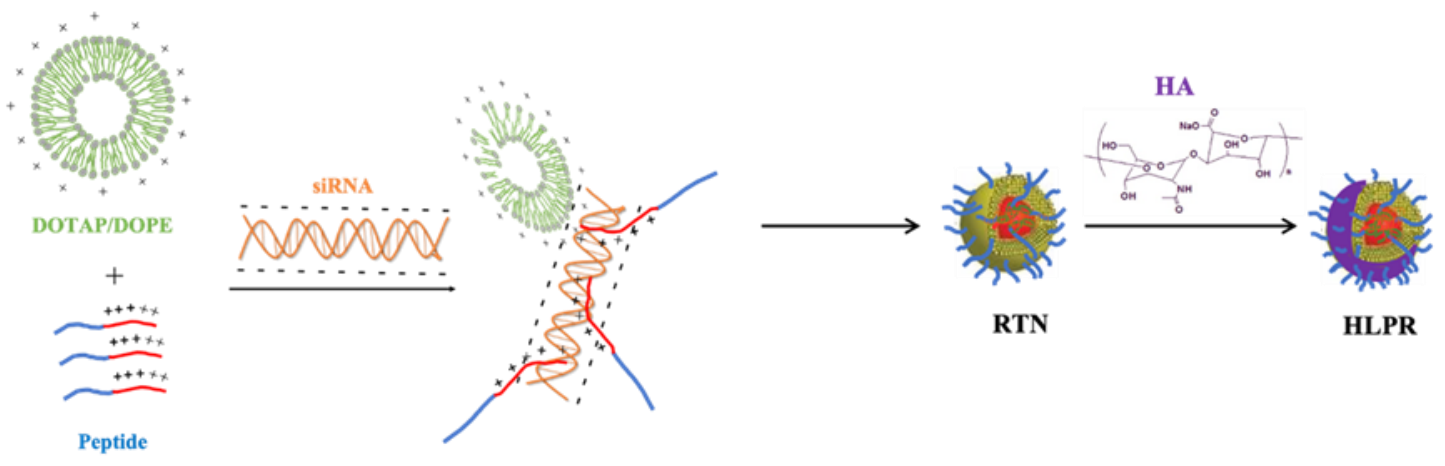

Figure 8 Illustration of the formation and structure of HA coated RTN. 


\subsubsection{Other strategies}

Modification of nanoparticles by applying albumin to the outer layer can also improve the stability of nanoparticles [82].

In short, improving the stability of the drug-carrier complex in the circulation is an important part in the design of delivery systems. If the drug is released before arriving at the lesion site, it will be quickly degraded and cleared, or even cause severe side effects. When it reaches the lesion site, if the drug dose is too low, not only will it have no therapeutic effects, but also may cause adverse reactions, such as the induction of drug resistance in tumor cells. Ideally, the drug delivery nanoparticles would enter the target cells in a complete form, but at the same time be released immediately to the target cells. Although an excessively-stable system shows good persistence in the circulation in vivo, it is meaningless if the drug cannot be released [83]. Therefore, mastering the balance between stability and release is the key to the nucleic acid delivery process.

\section{Targeted delivery}

As we know, apart from being unstable in the body, siRNA is not targeted before it enters the target cell. Patisiran, which has been approved by the FDA, and the potential carriers, such as Invivofectamine ${ }^{\mathrm{tm}}$ and in vivo-jet-PEI ${ }^{\mathrm{tm}}$, are all limited to liver delivery[84]. For tumor treatment, non-targeted drugs reach all parts of the body and attack all cells, leading to toxicity of the drug before the tumor is killed. Targeted delivery can not only reduce the toxic and side effects of the drug, but also allow more drugs to gather in the lesion area and increase the lethality of the drug. A large number of studies have also shown that active targeting is indispensable for improving drug efficacy and reducing side effects. 


\subsection{Environment-responsive delivery}

Targeting strategies are mainly divided into two categories: environmentallyresponsive delivery and receptor-mediated delivery. By exploiting the differences between the tumor microenvironment and normal tissues, researchers have designed a variety of stimulus-response, drug delivery systems. Strategies for internal environmental stimulus response includes $\mathrm{pH}$-responsive drug delivery systems (DDS) based on the acidic microenvironment of the tumor [85, 86], redox-sensitive DDS based on the reducing environment and enzyme-sensitive DDS based on overexpressed proteins in the tumor [87]. The external environment response includes the stimulations of light, heat, X-rays, and ultrasound, to achieve enhanced drug release at the target site. However, the effect of single-factor, response-based targeted design was found to be unsatisfactory in subsequent studies. For example, in ROS-responsive systems, extracellular ROS could cause premature drug release, and because of low ROS levels and uneven distribution in the tumor, inadequate responses can also occur [75]. Therefore, the designs of multiple environmental responses have been developed such as. a sequential response drug delivery system for siRNA delivery[75]. The nanoparticles contain a three-layer structure, the core layer of which is PEI600 combined with the photosensitizer rose bengal; the middle layer carries siRNA connected to the PEI by a singlet oxygen-sensitive double selenium bond, and the R8 cell penetrating peptide is modified onto it; the outer layer is covered by hyaluronic acid. Through $808 \mathrm{~nm}$ near-infrared irradiation, the core layer generates $540 \mathrm{~nm}$ radiation, which activates rose bengal to generate ROS. Then it promotes the degradation of double selenium bonds and releases the siRNA. Compared with the control group without rose red, the design showed obvious increased tumor suppression and promising potential application in precision medicine. 
In addition to the complex designs described above, HA-based nanocarriers can also be used for tumor microenvironment responsive delivery. Not only does the large amount of hyaluronidase existing outside the tumor cells degrade HA, the high level of intracellular concentration of ROS can also act on $\beta$ - $(1 \rightarrow 4)$ glycosidic bonds to break it down. What's more, HA is readily available and achieves multiple responses so is suitable for industrialization [87].

Designing a stimulus-responsive drug delivery system based on the tumor microenvironment increases the targeting of the drug to some extent. However, due to the heterogeneity of the tumor, the slight differences between the internal environment of tumors and normal tissue often leads to a failure of triggering the response from the nanocarriers due to lack of specificity and sensitivity. Understanding the best timing and specific location for the stimulus is also the key to efficient therapy by this approach.

\subsection{Receptor-mediated delivery}

Receptor-mediated drug delivery systems are one of the most important targeting strategies at present, and the presence of receptors overexpressed on the surface of the lesion cells provide opportunities for active targeted drug delivery.

The introduction of folic acid as a targeting group is one of the most widely used receptor targeting strategies in cancer therapy. Targeting cancer cells through folic acid can effectively improve drug targeting and reduce side effects [88].

In the treatment of inflammation by siRNA, T cells are difficult to transfect because of the lack of caveolae which leads to inactive endocytosis of nanoparticles. Fortunately, transferrin receptors are highly expressed in inflammatory tissues and cancer cells. As a transmembrane protein capable of specifically binding to transferrin, 
it can readily transfer iron into cells through receptor-mediated endocytosis. A PEIbased siRNA delivery strategy for cancer and inflammation treatment achieved targeting delivery by using transferrin, (reported by Kandil, Rima et al). Compared to pure PEI, the particle size was smaller and the zeta potential changed from positive to negative, but the nucleic acid loading efficiency was slightly lower than that of pure PEI, which may be because the steric hindrance of transferrin interferes with the binding between siRNA and PEI [89].

In recent years, antibodies have been used to modify carriers. On one hand, they are potential ligands for targeted delivery, and on the other hand, as a biological drug, they can also be used as synergistic treatments with siRNA [90]. Furthermore, aptamers also play a role in targeted delivery due to their advantages in chemical synthesis, and it is easier to perform quality control in subsequent studies, which leads to a growing interest in the study of aptamers [91].

\subsection{Other potential strategies}

In addition to receptor-mediated targeted delivery, the emergence of nanoantibodies opens a new door for targeted delivery of siRNA. With their unique chemical and biophysical properties, their specificity towards antigens can be improved via their non-canonical architectures, thereby increasing binding affinity. Nanoantibodies are stable, soluble, and show no signs of aggregation when produced alone, which is in contrast to original human antibodies. Compared with the toxic or immune response of other carriers, nanoantibodies are safer and less likely to cause an immune response due to the absence of effector regions $(\mathrm{Fc})$. According to recent research by Cunha-Santos, Catarina, etc., unlike the T-cell targeting strategy discussed above, the engineered nanoantibody targeting the CXCR4 receptor for the treatment of AIDS showed high efficiency of antibody-targeted delivery, which proved that siRNA 
delivery can still be achieved without using extra carriers [92].

The targeted delivery systems talked above were constructed by using substances which have a known target. Robert Langer's team synthesized a series of PEI-based low-molecular-weight vectors for siRNA delivery and selected 7C1 which possessed high transfection ability (needs reference). Based on previous studies, low molecular weight PEI was not suitable for siRNA delivery. However, by modification with alkyl chains, they produced a low-molecular-weight carrier with high efficiency and low toxicity for siRNA delivery. More interestingly, 7C1 specifically targets endothelial cells and has the potential to treat a variety of diseases caused by endothelial dysfunction [93]. Recently, the team published the data of 7C1 for siRNA delivery in non-human primates. Surprisingly, Tie2 siRNA loaded by $7 \mathrm{C} 1$ is preferentially delivered to endothelial cells of various organs in non-human primates, especially the lungs and heart, providing new evidence for endothelial gene silencing [94].

The development of targeted drug delivery systems has greatly reduced the side effects of drugs on non-focal sites, especially for anti-cancer drugs with high toxicity. In fact, all of the targeted strategies described only display a relative enhancement of targeting specificity, as the toxicity for normal sites cannot be avoided completely. For example, the over-expressed receptors of tumor cells may also be expressed in normal cells, but their affinity or quantity may be less than that of the lesion. In addition, the current carriers are not smart enough to reach the target site and only rely on blood flow to achieve drug delivery. If the drug carrier can rely on its own ability to reach the target site and bind to it specifically, a more effective drug carrier may be obtained.

\section{5. siRNA release from carriers}

After the vector carrying siRNA enters the cell through endocytosis, it has to be 
released into the cytoplasm or it will be degraded enzymes in the lysosome [95].Further, endosomes may pump them out through the ATP-bound cassette protein B1 when the nanoparticles enter the endosome [96]. In addition, a slow rate of drug release may lead to ineffective drug concentrations in target cells and promote resistance [97]. Therefore, achieving successful endosome escape is the key to the effectiveness of siRNA.

\subsection{Membrane fusion}

Generally, drugs enteringcells through endocytosis firstly encounters the primary endosome, then as the endosome matures to form lysosomes, the $\mathrm{pH}$ inside the endosome drops from 7.4 to 5.0. Therefore, based on the acidified environment, escape strategies can be designed [98]. We know that viruses exploit the acidic environment of the endosome to trigger protein transformation into an alpha helical conformation to promote membrane fusion, through which their contents are released [99]. Similarly, it is a good way to achieve drug release by incorporating substances such as influenza virus into the carrier. However, the toxicity of virus carriers can cause severe side effects, even death, so non-viral mimics of viral properties may be safer. Kanamala, Manju et al. designed an acid-triggered dePEGylation formulation, which achieved drug release via membrane fusion between liposomes and endosome [100]. In another case, DlinDMA was designed as a siRNA carrier but had a poor ability of endosome escape. During the modification process, the ether linker in DlinDMA was replaced by a ketal linker (DLin-KC2-DMA), which reduced the phase transition temperature. This modification promoted the formation of hexagonal structures when interacting with the endosomal membrane, enabling escape for drugs [101]. In addition, lipids also play an important role in endosomal escape. Previous studies on the function of helper lipids in liposomes had shown that, the hexagonal structure of fusion DOPE enhances endosomal escape, which is more effective than DOPC for gene transfection [102]. 


\subsection{Proton sponge effect}

Another mechanism of endosomal escape is called the proton sponge effect which was proposed in 1974 . When a carrier with a certain buffering capacity enters the acidic lysosome, it can consume large amounts of protons around it and promote the influx of chloride ions, which causes the endosome to swell and rupture due to the increased osmotic pressure. The buffering effect of the carrier, on the one hand, avoids an overacid endosome, on the other hand, it prolongs the residence time of the siRNA in the endosome and increases its chance to escape [103]. As for the lipid-derived carrier talked above, the hydrophobic chain of it must contains at least one tertiary amino group to enhance the buffering ability of materials and promote endosomal escape [104]. Han $\mathrm{K}$ et al. used an amphiphilic peptide (Fmoc) 2KH7-TAT containing seven arginines to load doxorubicin and DNA. As it can be protonated more extensively than formulations without arginine, 2KH7-TAT prevents the acidification process caused by ATPase to a certain extent, but also improves the osmotic pressure of the endosome which helps endosome escape of the drug [85]. Based on this, Li GR designed a dual immune checkpoint suppression strategy, which was formulated by loading PD-L1 siRNA and indoleamine 2,3-dioxidase inhibitor with transmembrane peptide Lin TT1. Arginine favorable for cell membrane penetration was also contained in this carrier [105]. The stable core-shell structure exhibits excellent endosome escape ability [95].

However, this theory has not been proven yet, and there are still many controversies [103]. Similarly, there are other strategies that are also based on the principle of endosome swelling but employing mechanical disruption rather than osmotic pressure. Lee SH et al. prepared Pluronic/PEI nano capsules, through a short cold shock at $15^{\circ} \mathrm{C}$ to make the temperature-sensitive Pluronic expand to promote endosome escape [106]. But unlike other delivery systems based on internal 
environmental stimuli, this method requires dedicated, specific equipment and must takes both time and space into account to control the timing of the stimulus. If the carrier swells in advance, not only is it no help with escape from the endosome, but also prevents it from entering the cell.

\subsection{Membrane pore formation}

The third mechanism is called membrane pore formation based on the interaction between the membrane tension of the enlarged pores and the linear tension of the closed pores. Some peptides have a good affinity for the rims of such pores. Their combination leads to a reduction in line tension and forms a pores with stable radius on the membrane, further providing the endosome contents with opportunity to escape [107].

\subsection{Photochemical disruption}

Photosensitizers can produce reactive singlet oxygen under light stimulation to destroy the endosome membrane, which can also mediate the escape of siRNA, and it will not affect the drug as the life of reactive singlet oxygen is too short to destroy the structural integrity of siRNA [108].

\section{Summary}

Among the numerous therapeutic strategies in the field of gene therapy, siRNA has won a place due to its unique properties. Compared to pDNA, siRNA has a lower molecular weight, consisting of only 20-30 base pairs, and does not require delivery to the nucleus while siRNA is more specific than microRNA. However, the different nature of siRNA compared to pDNA means that siRNA often cannot be delivered with the same formulations. To achieve a successful deliver siRNA in vivo, it is critical to 
develop the design of specific vectors optimized for siRNA.

For the moment, lipid-based and polymer-based carriers have been widely studied due to their good delivery efficiency, but there are still shortcomings that need to be improved, including stability, targeting ability, and endosomal release. In response to these problems, researchers have made efforts to optimize these carriers, such as imitating the viral protein structures and functions and establishing the relation between molecular structure and delivery efficiency. Based on the current mechanism of endosomal escape of siRNA, a buffering group or membrane fusion substance is introduced into the vector. In terms of reducing off-target effects of drugs, further exploration of high-efficiency specific carriers is needed. In addition, advanced techniques for characterization still need to be developed continuously to support related research.

So far, two siRNA drugs have come to market, marking the era of siRNA. The acceleration of research on siRNA delivery vectors will have great significance for siRNA in clinical treatment.

\section{Acknowledges}

This work was supported by the National Natural Science Foundation of China (No. 81690262) and Minhang "Production-Study-Research" Project in Sanghai (No. 2019MH229). SH is supported by the NIHR Great Ormond Street Hospital Biomedical Research Centre

\section{References}

[1] D. Bumcrot, M. Manoharan, V. Koteliansky, D.W.Y. Sah, RNAi therapeutics: a potential new class of pharmaceutical drugs, Nat. Chem. Biol. 2(12) (2006) 711-719.

[2] M. Sako, F.R. Song, A. Okamoto, H. Koide, T. Dewa, N. Oku, T. Asai, Key determinants of siRNA delivery mediated by unique pH-responsive lipid-based liposomes, Int. J. Pharm. 569 (2019) 12.

[3] B. Kim, J.H. Park, M.J. Sailor, Rekindling RNAi Therapy: Materials Design Requirements for In Vivo siRNA Delivery, Adv Mater (2019) e1903637. 
[4] C. Baum, O. Kustikova, U. Modlich, Z. Li, B. Fehse, Mutagenesis and oncogenesis by chromosomal insertion of gene transfer vectors, Hum. Gene Ther. 17(3) (2006) 253-63.

[5] N. Bessis, F.J. GarciaCozar, M.C. Boissier, Immune responses to gene therapy vectors: influence on vector function and effector mechanisms, Gene Ther. 11 Suppl 1 (2004) S10-7.

[6] N. Somia, I.M. Verma, Gene therapy: trials and tribulations, Nat Rev Genet 1(2) (2000) 91-9.

[7] C.E. Thomas, A. Ehrhardt, M.A. Kay, Progress and problems with the use of viral vectors for gene therapy, Nat. Rev. Genet. 4(5) (2003) 346-58.

[8] D. Bouard, D. Alazard-Dany, F.L. Cosset, Viral vectors: from virology to transgene expression, Br. J. Pharmacol. 157(2) (2009) 153-65.

[9] S. Zhang, B. Zhao, H. Jiang, B. Wang, B. Ma, Cationic lipids and polymers mediated vectors for delivery of siRNA, J Control Release 123(1) (2007) 1-10.

[10] J. Jiang, X. Zhang, Y. Tang, S. Li, J. Chen, Progress on ocular siRNA gene-silencing therapy and drug delivery systems, Fundam Clin Pharmacol (2020).

[11] F.F. Yang, W. Huang, Y.F. Li, Z.G. Gao, [Current status of non-viral vectors for siRNA delivery], Yao Xue Xue Bao 46(12) (2011) 1436-43.

[12] P.R. de Paula Brandao, S.S. Titze-de-Almeida, R. Titze-de-Almeida, Leading RNA Interference Therapeutics Part 2: Silencing Delta-Aminolevulinic Acid Synthase 1, with a Focus on Givosiran, Mol Diagn Ther (2019).

[13] H. Yin, R.L. Kanasty, A.A. Eltoukhy, A.J. Vegas, J.R. Dorkin, D.G. Anderson, Non-viral vectors for gene-based therapy, Nat. Rev. Genet. 15(8) (2014) 541-55.

[14] K. Buyens, B. Lucas, K. Raemdonck, K. Braeckmans, J. Vercammen, J. Hendrix, Y. Engelborghs, S.C. De Smedt, N.N. Sanders, A fast and sensitive method for measuring the integrity of siRNA-carrier complexes in full human serum, J. Controlled Release 126(1) (2008) 67-76.

[15] E. Nogueira, J. Freitas, A. Loureiro, P. Nogueira, A.C. Gomes, A. Preto, A.M. Carmo, A. Moreira, A. Cavaco-Paulo, Neutral PEGylated liposomal formulation for efficient folate-mediated delivery of MCL1 siRNA to activated macrophages, Colloids Surf. B. Biointerfaces 155 (2017) 459-465.

[16] J.A. Kulkarni, P.R. Cullis, R. van der Meel, Lipid Nanoparticles Enabling Gene Therapies: From Concepts to Clinical Utility, Nucleic Acid Ther 28(3) (2018) 146-157.

[17] H.R. Bender, S. Kane, M.D. Zabel, Delivery of Therapeutic siRNA to the CNS Using Cationic and Anionic Liposomes, J Vis Exp (113) (2016).

[18] D.A. Balazs, W. Godbey, Liposomes for use in gene delivery, J Drug Deliv 2011 (2011) 326497.

[19] M.A. Maier, M. Jayaraman, S. Matsuda, J. Liu, S. Barros, W. Querbes, Y.K. Tam, S.M. Ansell, V. Kumar, J. Qin, X.M. Zhang, Q.F. Wang, S. Panesar, R. Hutabarat, M. Carioto, J. Hettinger, P. Kandasamy, D. Butler, K.G. Rajeev, B. Pang, K. Charisse, K. Fitzgerald, B.L. Mui, X.Y. Du, P. Cullis, T.D. Madden, M.J. Hope, M. Manoharan, A. Akinc, Biodegradable Lipids Enabling Rapidly Eliminated Lipid Nanoparticles for Systemic Delivery of RNAi Therapeutics, Mol. Ther. 21(8) (2013) 1570-1578.

[20] J. Lee, P.E. Saw, V. Gujrati, Y. Lee, H. Kim, S. Kang, M. Choi, J.-I. Kim, S. Jon, Mono-arginine Cholesterol-based Small Lipid Nanoparticles as a Systemic siRNA Delivery Platform for Effective Cancer Therapy, Theranostics 6(2) (2016) 192-203.

[21] G. Sahay, W. Querbes, C. Alabi, A. Eltoukhy, S. Sarkar, C. Zurenko, E. Karagiannis, K. Love, D.L. Chen, R. Zoncu, Y. Buganim, A. Schroeder, R. Langer, D.G. Anderson, Efficiency of siRNA delivery by lipid nanoparticles is limited by endocytic recycling, Nat. Biotechnol. 31(7) (2013) 653-U119. 
[22] C.A. Alabi, K.T. Love, G. Sahay, H. Yin, K.M. Luly, R. Langer, D.G. Anderson, Multiparametric approach for the evaluation of lipid nanoparticles for siRNA delivery, Proceedings of the National Academy of Sciences of the United States of America 110(32) (2013) 12881-12886.

[23] K.K. Ewert, A. Ahmad, H.M. Evans, C.R. Safinya, Cationic lipid-DNA complexes for non-viral gene therapy: relating supramolecular structures to cellular pathways, Expert Opin Biol Ther 5(1) (2005) 3353.

[24] S.C. Semple, A. Akinc, J. Chen, A.P. Sandhu, B.L. Mui, C.K. Cho, D.W. Sah, D. Stebbing, E.J. Crosley, E. Yaworski, I.M. Hafez, J.R. Dorkin, J. Qin, K. Lam, K.G. Rajeev, K.F. Wong, L.B. Jeffs, L. Nechev, M.L. Eisenhardt, M. Jayaraman, M. Kazem, M.A. Maier, M. Srinivasulu, M.J. Weinstein, Q. Chen, R. Alvarez, S.A. Barros, S. De, S.K. Klimuk, T. Borland, V. Kosovrasti, W.L. Cantley, Y.K. Tam, M. Manoharan, M.A. Ciufolini, M.A. Tracy, A. de Fougerolles, I. MacLachlan, P.R. Cullis, T.D. Madden, M.J. Hope, Rational design of cationic lipids for siRNA delivery, Nat. Biotechnol. 28(2) (2010) 172-6.

[25] K.K. Ewert, A. Ahmad, H.M. Evans, C.R. Safinya, Cationic lipid-DNA complexes for non-viral gene therapy: relating supramolecular structures to cellular pathways, Expert Opin Biol Th 5(1) (2005) 33-53. [26] F. Cardarelli, D. Pozzi, A. Bifone, C. Marchini, G. Caracciolo, Cholesterol-dependent macropinocytosis and endosomal escape control the transfection efficiency of lipoplexes in CHO living cells, Mol. Pharm. 9(2) (2012) 334-40.

[27] F. Song, N. Sakurai, A. Okamoto, H. Koide, N. Oku, T. Dewa, T. Asai, Design of a Novel PEGylated Liposomal Vector for Systemic Delivery of siRNA to Solid Tumors, Biol. Pharm. Bull. 42(6) (2019) 9961003.

[28] A.C.N. Oliveira, J. Fernandes, A. Goncalves, A.C. Gomes, M. Oliveira, Lipid-based Nanocarriers for siRNA Delivery: Challenges, Strategies and the Lessons Learned from the DODAX: MO Liposomal System, Curr Drug Targets 20(1) (2019) 29-50.

[29] Y. Xia, J. Tian, X. Chen, Effect of surface properties on liposomal siRNA delivery, Biomaterials 79 (2016) 56-68.

[30] F. Haghiralsadat, G. Amoabediny, S. Naderinezhad, T. Forouzanfar, M.N. Helder, B. Zandieh-Doulabi, Preparation of PEGylated cationic nanoliposome-siRNA complexes for cancer therapy, Artif Cells Nanomed Biotechnol 46(sup1) (2018) 684-692.

[31] R.K. Fisher, S.I. Mattern-Schain, M.D. Best, S.S. Kirkpatrick, M.B. Freeman, O.H. Grandas, D.J.H. Mountain, Improving the efficacy of liposome-mediated vascular gene therapy via lipid surface modifications, J Surg Res 219 (2017) 136-144.

[32] T. Tagami, T. Suzuki, M. Matsunaga, K. Nakamura, N. Moriyoshi, T. Ishida, H. Kiwada, Antiangiogenic therapy via cationic liposome-mediated systemic siRNA delivery, Int J Pharm 422(1-2) (2012) 280-9.

[33] H.-L. Jiang, M.A. Islam, L. Xing, J. Firdous, W. Cao, Y.-J. He, Y. Zhu, K.-H. Cho, H.-S. Li, C.-S. Cho, Degradable Polyethylenimine-Based Gene Carriers for Cancer Therapy, in: Y. Cheng (Ed.), Polymeric Gene Delivery Systems, Springer International Publishing Ag, Gewerbestrasse 11, Cham, Ch-6330, Switzerland2018, pp. 113-148.

[34] J. Luten, C.F. van Nostrum, S.C. De Smedt, W.E. Hennink, Biodegradable polymers as non-viral carriers for plasmid DNA delivery, J. Controlled Release 126(2) (2008) 97-110.

[35] K. Buyens, M. Meyer, E. Wagner, J. Demeester, S.C. De Smedt, N.N. Sanders, Monitoring the disassembly of siRNA polyplexes in serum is crucial for predicting their biological efficacy, J Control 
Release 141(1) (2010) 38-41.

[36] J. Zhou, J. Wu, N. Hafdi, J.P. Behr, P. Erbacher, L. Peng, PAMAM dendrimers for efficient siRNA delivery and potent gene silencing, Chem Commun (Camb) (22) (2006) 2362-4.

[37] H.J. Kim, K. Miyata, T. Nomoto, M. Zheng, A. Kim, X. Liu, H. Cabral, R.J. Christie, N. Nishiyama, K. Kataoka, siRNA delivery from triblock copolymer micelles with spatially-ordered compartments of PEG shell, siRNA-loaded intermediate layer, and hydrophobic core, Biomaterials 35(15) (2014) 4548-56.

[38] A. Gonsho, K. Irie, H. Susaki, H. Iwasawa, S. Okuno, T. Sugawara, Tissue-targeting ability of saccharide-poly(L-lysine) conjugates, Biol Pharm Bull 17(2) (1994) 275-82.

[39] Y. Cao, Y.F. Tan, Y.S. Wong, M.W.J. Liew, S. Venkatraman, Recent Advances in Chitosan-Based Carriers for Gene Delivery, Mar Drugs 17(6) (2019).

[40] Z. Du, M. Chen, Q. He, Y. Zhou, T. Jin, Polymerized spermine as a novel polycationic nucleic acid carrier system, Int J Pharm 434(1-2) (2012) 437-43.

[41] Z. Du, S. Xiang, Y. Zang, Y. Zhou, C. Wang, H. Tang, T. Jin, X. Zhang, Polyspermine imine, a pH responsive polycationic siRNA carrier degradable to endogenous metabolites, Mol Pharm 11(10) (2014) 3300-6.

[42] Y. Liu, J. Chen, Y. Tang, S. Li, Y. Dou, J. Zheng, Synthesis and Characterization of Quaternized Poly( $\beta$ amino ester) for Highly Efficient Delivery of Small Interfering RNA, Mol. Pharm. 15(10) (2018) 45584567.

[43] M.L. Patil, M. Zhang, T. Minko, Multifunctional triblock Nanocarrier (PAMAM-PEG-PLL) for the efficient intracellular siRNA delivery and gene silencing, ACS Nano 5(3) (2011) 1877-87.

[44] J. Kim, Y. Kang, S.Y. Tzeng, J.J. Green, Synthesis and application of poly(ethylene glycol)-co-poly( $\beta$ amino ester) copolymers for small cell lung cancer gene therapy, Acta Biomater. 41 (2016) 293-301.

[45] X. Sun, N. Zhang, Cationic polymer optimization for efficient gene delivery, Mini Rev Med Chem 10(2) (2010) 108-25.

[46] Y. Hayashi, T. Higashi, K. Motoyama, H. Jono, Y. Ando, R. Onodera, H. Arima, Hepatocyte-Targeted Delivery of siRNA Polyplex with PEG-Modified Lactosylated Dendrimer/Cyclodextrin Conjugates for Transthyretin-Related Amyloidosis Therapy, Biol Pharm Bull 42(10) (2019) 1679-1688.

[47] D. Ulkoski, A. Bak, J.T. Wilson, V.R. Krishnamurthy, Recent advances in polymeric materials for the delivery of RNA therapeutics, Expert Opin Drug Deliv 16(11) (2019) 1149-1167.

[48] O. Boussif, F. Lezoualc'h, M.A. Zanta, M.D. Mergny, D. Scherman, B. Demeneix, J.P. Behr, A versatile vector for gene and oligonucleotide transfer into cells in culture and in vivo: polyethylenimine, Proc Natl Acad Sci U S A 92(16) (1995) 7297-301.

[49] S.D. Li, L. Huang, Nanoparticles evading the reticuloendothelial system: role of the supported bilayer, Biochim Biophys Acta 1788(10) (2009) 2259-66.

[50] Y. Tan, M. Whitmore, S. Li, P. Frederik, L. Huang, LPD nanoparticles--novel nonviral vector for efficient gene delivery, Methods Mol Med 69 (2002) 73-81.

[51] D.P. Vangasseri, S.J. Han, L. Huang, Lipid-protamine-DNA-mediated antigen delivery, Curr Drug Deliv 2(4) (2005) 401-6.

[52] Q.F. Yu, B. Zhang, Y.L. Zhou, Q. Ge, J.L. Chang, Y.N. Chen, K.Q. Zhang, D.Y. Peng, W.D. Chen, Codelivery of gambogenic acid and VEGF-siRNA with anionic liposome and polyethylenimine complexes to HepG2 cells, J. Liposome Res. 29(4) (2019) 322-331.

[53] A.D. Tagalakis, D.H.D. Lee, A.S. Bienemann, H.Y. Zhou, M.M. Munye, L. Saraiva, D. McCarthy, Z.X. 
Du, C.A. Vink, R. Maeshima, E.A. White, K. Gustafsson, S.L. Hart, Multifunctional, self-assembling anionic peptide-lipid nanocomplexes for targeted siRNA delivery, Biomaterials 35(29) (2014) 8406-8415.

[54] S.L. Hart, Multifunctional nanocomplexes for gene transfer and gene therapy, Cell Biol Toxicol 26(1) (2010) 69-81.

[55] G.D. Kenny, C. Villegas-Llerena, A.D. Tagalakis, F. Campbell, K. Welser, M. Botta, A.B. Tabor, H.C. Hailes, M.F. Lythgoe, S.L. Hart, Multifunctional receptor-targeted nanocomplexes for magnetic resonance imaging and transfection of tumours, Biomaterials 33(29) (2012) 7241-50.

[56] M. Rezaee, R.K. Oskuee, H. Nassirli, B. Malaekeh-Nikouei, Progress in the development of lipopolyplexes as efficient non-viral gene delivery systems, J Control Release 236 (2016) 1-14.

[57] S.R. Pinnapireddy, L. Duse, B. Strehlow, J. Schäfer, U. Bakowsky, Composite liposome-PEI/nucleic acid lipopolyplexes for safe and efficient gene delivery and gene knockdown, Colloids Surf. B. Biointerfaces 158 (2017) 93-101.

[58] S. Santiwarangkool, H. Akita, I.A. Khalil, M.M. Abd Elwakil, Y. Sato, K. Kusumoto, H. Harashima, A study of the endocytosis mechanism and transendothelial activity of lung-targeted GALA-modified liposomes, J Control Release 307 (2019) 55-63.

[59] J. Lee, H.J. Ahn, PEGylated DC-Chol/DOPE cationic liposomes containing KSP siRNA as a systemic siRNA delivery Carrier for ovarian cancer therapy, Biochem. Biophys. Res. Commun. 503(3) (2018) 17161722.

[60] T. Ishitsuka, H. Akita, H. Harashima, Functional improvement of an IRQ-PEG-MEND for delivering genes to the lung, J Control Release 154(1) (2011) 77-83.

[61] K. Hashiba, Y. Sato, H. Harashima, pH-labile PEGylation of siRNA-loaded lipid nanoparticle improves active targeting and gene silencing activity in hepatocytes, J Control Release 262 (2017) 239-246.

[62] T.H. Kim, Y.G. Jo, H.H. Jiang, S.M. Lim, Y.S. Youn, S. Lee, X. Chen, Y. Byun, K.C. Lee, PEG-transferrin conjugated TRAIL (TNF-related apoptosis-inducing ligand) for therapeutic tumor targeting, J. Controlled Release 162(2) (2012) 422-428.

[63] C.A. Hurley, J.B. Wong, J. Ho, M. Writer, S.A. Irvine, M.J. Lawrence, S.L. Hart, A.B. Tabor, H.C. Hailes, Mono- and dicationic short PEG and methylene dioxyalkylglycerols for use in synthetic gene delivery systems, Org Biomol Chem 6(14) (2008) 2554-9.

[64] A.D. Tagalakis, S.M. Grosse, Q.H. Meng, M.F. Mustapa, A. Kwok, S.E. Salehi, A.B. Tabor, H.C. Hailes, S.L. Hart, Integrin-targeted nanocomplexes for tumour specific delivery and therapy by systemic administration, Biomaterials 32(5) (2011) 1370-6.

[65] G. Cavallaro, C. Sardo, E.F. Craparo, B. Porsio, G. Giammona, Polymeric nanoparticles for siRNA delivery: Production and applications, Int. J. Pharm. 525(2) (2017) 313-333.

[66] M.X. Zhou, H. Huang, D.Q. Wang, H.R. Lu, J. Chen, Z.F. Chai, S.Q. Yao, Y. Hu, Light-Triggered PEGylation/dePEGylation of the Nanocarriers for Enhanced Tumor Penetration, Nano Lett. 19(6) (2019) 3671-3675.

[67] M. Kanamala, B.D. Palmer, W.R. Wilson, Z.M. Wu, Characterization of a smart pH-cleavable PEG polymer towards the development of dual pH-sensitive liposomes, Int. J. Pharm. 548(1) (2018) 288-296. [68] A.S. Nosova, O.O. Koloskova, A.A. Nikonova, V.A. Simonova, V.V. Smirnov, D. Kudlay, M.R. Khaitov, Diversity of PEGylation methods of liposomes and their influence on RNA delivery, Medchemcomm 10(3) (2019) 369-377.

[69] T. Ishida, M. Ichihara, X. Wang, K. Yamamoto, J. Kimura, E. Majima, H. Kiwada, Injection of 
PEGylated liposomes in rats elicits PEG-specific IgM, which is responsible for rapid elimination of a second dose of PEGylated liposomes, J. Controlled Release 112(1) (2006) 15-25.

[70] H. Hatakeyama, H. Akita, E. Ito, Y. Hayashi, M. Oishi, Y. Nagasaki, R. Danev, K. Nagayama, N. Kaji, H. Kikuchi, Y. Baba, H. Harashima, Systemic delivery of siRNA to tumors using a lipid nanoparticle containing a tumor-specific cleavable PEG-lipid, Biomaterials 32(18) (2011) 4306-4316.

[71] M. Tang, H.Q. Dong, Y.Y. Li, T.B. Ren, Harnessing the PEG-cleavable strategy to balance cytotoxicity, intracellular release and the therapeutic effect of dendrigraft poly-L-lysine for cancer gene therapy, Journal of Materials Chemistry B 4(7) (2016) 1284-1295.

[72] M. Zhou, H. Huang, D. Wang, H. Lu, J. Chen, Z. Chai, S.Q. Yao, Y. Hu, Light-Triggered PEGylation/dePEGylation of the Nanocarriers for Enhanced Tumor Penetration, Nano Lett. 19(6) (2019) 3671-3675.

[73] G.L. Huang, H.L. Huang, Hyaluronic acid-based biopharmaceutical delivery and tumor-targeted drug delivery system, J. Controlled Release 278 (2018) 122-126.

[74] I. Serrano-Sevilla, A. Artiga, S.G. Mitchell, L. De Matteis, J.M. de la Fuente, Natural Polysaccharides for siRNA Delivery: Nanocarriers Based on Chitosan, Hyaluronic Acid, and Their Derivatives, Molecules 24(14) (2019) 34.

[75] Y. He, S. Guo, L. Wu, P. Chen, L. Wang, Y. Liu, H. Ju, Near-infrared boosted ROS responsive siRNA delivery and cancer therapy with sequentially peeled upconversion nano-onions, Biomaterials 225 (2019) 119501.

[76] G. Jiang, K. Park, J. Kim, K.S. Kim, S.K. Hahn, Target specific intracellular delivery of siRNA/PEI-HA complex by receptor mediated endocytosis, Mol. Pharm. 6(3) (2009) 727-37.

[77] M.Y. Lee, S.J. Park, K. Park, K.S. Kim, H. Lee, S.K. Hahn, Target-specific gene silencing of layer-bylayer assembled gold-cysteamine/siRNA/PEI/HA nanocomplex, ACS Nano 5(8) (2011) 6138-47.

[78] K. Park, S.W. Hong, W. Hur, M.Y. Lee, J.A. Yang, S.W. Kim, S.K. Yoon, S.K. Hahn, Target specific systemic delivery of TGF- $\beta$ siRNA/(PEI-SS)-g-HA complex for the treatment of liver cirrhosis, Biomaterials 32(21) (2011) 4951-8.

[79] Y.Y. Liang, J.H. Peng, N. Li, C. Yu-Wai-Man, Q. Wang, Y.H. Xu, H.X. Wang, A.D. Tagalakis, Z.X. Du, Smart nanoparticles assembled by endogenous molecules for siRNA delivery and cancer therapy via CD44 and EGFR dual-targeting, Nanomedicine-Nanotechnology Biology and Medicine 15(1) (2019) 208217.

[80] F. Xie, L.C. Zhang, J.L. Peng, C. Lit, J. Pu, Y.H. Xu, Z.X. Du, Hepatic Carcinoma Selective Nucleic Acid Nanovector Assembled by Endogenous Molecules Based on Modular Strategy, Mol. Pharm. 14(6) (2017) 1841-1851.

[81] Y. Shen, B. Wang, Y. Lu, A. Ouahab, Q. Li, J. Tu, A novel tumor-targeted delivery system with hydrophobized hyaluronic acid-spermine conjugates (HHSCs) for efficient receptor-mediated siRNA delivery, Int. J. Pharm. 414(1) (2011) 233-243.

[82] J.J. Dang, H. Ye, Y.J. Li, Q.J. Liang, X.D. Li, L.C. Yin, Multivalency-assisted membrane-penetrating SiRNA delivery sensitizes photothermal ablation via inhibition of tumor glycolysis metabolism, Biomaterials 223 (2019) 14.

[83] Y.Y. Wang, M.Z. Ye, R.S. Xie, S.Q. Gong, Enhancing the In Vitro and In Vivo Stabilities of Polymeric Nucleic Acid Delivery Nanosystems, Bioconj. Chem. 30(2) (2019) 325-337.

[84] N. Raval, H. Jogi, P. Gondaliya, K. Kalia, R.K. Tekade, Method and its Composition for encapsulation, 
stabilization, and delivery of siRNA in Anionic polymeric nanoplex: An In vitro- In vivo Assessment, Scientific reports 9(1) (2019) 16047.

[85] K. Han, S. Chen, W.-H. Chen, Q. Lei, Y. Liu, R.-X. Zhuo, X.-Z. Zhang, Synergistic gene and drug tumor therapy using a chimeric peptide, Biomaterials 34(19) (2013) 4680-4689.

[86] H.L. Tang, W.L. Zhao, J.M. Yu, Y. Li, C. Zhao, Recent Development of pH-Responsive Polymers for Cancer Nanomedicine, Molecules 24(1) (2019) 24.

[87] K.Y. Choi, H.S. Han, E.S. Lee, J.M. Shin, B.D. Almquist, D.S. Lee, J.H. Park, Hyaluronic Acid-Based Activatable Nanomaterials for Stimuli-Responsive Imaging and Therapeutics: Beyond CD44-Mediated Drug Delivery, Advanced Materials 31(34) (2019) 18.

[88] L. Liu, M. Zheng, D. Librizzi, T. Renette, O.M. Merkel, T. Kissel, Efficient and Tumor Targeted siRNA Delivery by Polyethylenimine-graft-polycaprolactone-block-poly(ethylene glycol)-folate (PEI-PCL-PEGFol), Mol. Pharm. 13(1) (2016) 134-143.

[89] R. Kandil, Y. Xie, A. Mehta, O. Merkel, A Method for Targeted Nonviral siRNA Delivery in Cancer and Inflammatory Diseases, Methods in molecular biology (Clifton, N.J.) 2059 (2020) 155-166.

[90] J. Zhao, Y. Mi, S.-S. Feng, Targeted co-delivery of docetaxel and siPlk1 by herceptin-conjugated vitamin E TPGS based immunomicelles, Biomaterials 34(13) (2013) 3411-3421.

[91] T.C. Chu, K.Y. Twu, A.D. Ellington, M. Levy, Aptamer mediated siRNA delivery, Nucleic Acids Res. 34(10) (2006) e73-e73.

[92] C. Cunha-Santos, P.R.L. Perdigao, F. Martin, J.G. Oliveira, M. Cardoso, A. Manuel, N. Taveira, J. Goncalves, Inhibition of HIV replication through siRNA carried by CXCR4-targeted chimeric nanobody, Cell. Mol. Life Sci. (2019).

[93] J.E. Dahlman, C. Barnes, O.F. Khan, A. Thiriot, S. Jhunjunwala, T.E. Shaw, Y.P. Xing, H.B. Sager, G. Sahay, L. Speciner, A. Bader, R.L. Bogorad, H. Yin, T. Racie, Y.Z. Dong, S. Jiang, D. Seedorf, A. Dave, K.S. Sandhu, M.J. Webber, T. Novobrantseva, V.M. Ruda, A.K.R. Lytton-Jean, C.G. Levins, B. Kalish, D.K. Mudge, M. Perez, L. Abezgauz, P. Dutta, L. Smith, K. Charisse, M.W. Kieran, K. Fitzgerald, M. Nahrendorf, D. Danino, R.M. Tuder, U.H. von Andrian, A. Akinc, D. Panigrahy, A. Schroeder, V. Koteliansky, R. Langer, D.G. Anderson, In vivo endothelial siRNA delivery using polymeric nanoparticles with low molecular weight, Nature Nanotechnology 9(8) (2014) 648-655.

[94] O.F. Khan, P.S. Kowalski, J.C. Doloff, J.K. Tsosie, V. Bakthavatchalu, C.B. Winn, J. Haupt, M. Jamiel, R. Langer, D.G. Anderson, Endothelial siRNA delivery in nonhuman primates using ionizable lowmolecular weight polymeric nanoparticles, Science Advances 4(6) (2018) 10.

[95] G. Li, Y. Gao, C. Gong, Z. Han, L. Qiang, Z. Tai, J. Tian, S. Gao, Dual-Blockade Immune Checkpoint for Breast Cancer Treatment Based on a Tumor-Penetrating Peptide Assembling Nanoparticle, ACS applied materials \& interfaces 11(43) (2019) 39513-39524.

[96] Y.H. Yang, Z. Wang, Y. Peng, J.S. Ding, W.H. Zhou, A Smart pH-Sensitive Delivery System for Enhanced Anticancer Efficacy via Paclitaxel Endosomal Escape, Frontiers in Pharmacology 10 (2019) 11.

[97] H. Wu, L. Zhu, V.P. Torchilin, pH-sensitive poly(histidine)-PEG/DSPE-PEG co-polymer micelles for cytosolic drug delivery, Biomaterials 34(4) (2013) 1213-1222.

[98] S.A. Smith, L.I. Selby, A.P.R. Johnston, G.K. Such, The Endosomal Escape of Nanoparticles: Toward More Efficient Cellular Delivery, Bioconj. Chem. 30(2) (2019) 263-272.

[99] P.J. Klasse, R. Bron, M. Marsh, Mechanisms of enveloped virus entry into animal cells, Adv. Drug Del. Rev. 34(1) (1998) 65-91. 
[100] M. Kanamala, B.D. Palmer, S.M.F. Jamieson, W.R. Wilson, Z.M. Wu, Dual pH-sensitive liposomes with low pH-triggered sheddable PEG for enhanced tumor-targeted drug delivery, Nanomedicine 14(15) (2019) 1972-1990.

[101] T.M. Allen, P.R. Cullis, Liposomal drug delivery systems: From concept to clinical applications, Adv. Drug Del. Rev. 65(1) (2013) 36-48.

[102] Z.X. Du, M.M. Munye, A.D. Tagalakis, M.D.I. Manunta, S.L. Hart, The Role of the Helper Lipid on the DNA Transfection Efficiency of Lipopolyplex Formulations, Scientific Reports 4 (2014) 6.

[103] C.W. Pouton, L.W. Seymour, Key issues in non-viral gene delivery1PII of original article: S0169409X(98)00048-9. The article was originally published in Advanced Drug Delivery Reviews 34 (1998) 319.1, Adv. Drug Del. Rev. 46(1) (2001) 187-203.

[104] K.A. Whitehead, J.R. Dorkin, A.J. Vegas, P.H. Chang, O. Veiseh, J. Matthews, O.S. Fenton, Y.L. Zhang, K.T. Olejnik, V. Yesilyurt, D.L. Chen, S. Barros, B. Klebanov, T. Novobrantseva, R. Langer, D.G. Anderson, Degradable lipid nanoparticles with predictable in vivo siRNA delivery activity, Nature Communications 5 (2014) 10.

[105] J.K. Xu, A.R. Khan, M.F. Fu, R.J. Wang, J.B. Ji, G.X. Zhai, Cell-penetrating peptide: a means of breaking through the physiological barriers of different tissues and organs, J. Controlled Release 309 (2019) 106-124.

[106] S.H. Lee, S.H. Choi, S.H. Kim, T.G. Park, Thermally sensitive cationic polymer nanocapsules for specific cytosolic delivery and efficient gene silencing of siRNA: Swelling induced physical disruption of endosome by cold shock, J. Controlled Release 125(1) (2008) 25-32.

[107] H.W. Huang, F.Y. Chen, M.T. Lee, Molecular mechanism of Peptide-induced pores in membranes, Phys Rev Lett 92(19) (2004) 198304.

[108] A.K. Varkouhi, M. Scholte, G. Storm, H.J. Haisma, Endosomal escape pathways for delivery of biologicals, J. Controlled Release 151(3) (2011) 220-228. 